\title{
Subcarrier Index Coordinate Expression (SICE): An Ultra-low-power OFDM-compatible Wireless Communications Scheme Tailored for Internet of Things
}

\section{Citation}

Kuo P-H, Kung HT. 2014. "Subcarrier Index Coordinate Expression (SICE): An Ultra-low-power OFDM-compatible Wireless Communications Scheme Tailored for Internet of Things." Presented at the 4th International Conference on the Internet of Things (IoT 2014), Cambridge, MA, 3-8 October 2014.

\section{Published Version}

10.1109/IOT.2014.7030122

\section{Permanent link}

http://nrs.harvard.edu/urn-3:HUL.InstRepos:12841973

\section{Terms of Use}

This article was downloaded from Harvard University's DASH repository, and is made available under the terms and conditions applicable to Open Access Policy Articles, as set forth at http:// nrs.harvard.edu/urn-3:HUL.InstRepos:dash.current.terms-of-use\#OAP

\section{Share Your Story}

The Harvard community has made this article openly available.

Please share how this access benefits you. Submit a story. 


\title{
Subcarrier Index Coordinate Expression (SICE): An Ultra-low-power OFDM-compatible Wireless Communications Scheme Tailored for Internet of Things
}

\author{
Ping-Heng Kuo ${ }^{1,2}$ H. T. Kung ${ }^{1}$ \\ ${ }^{1}$ School of Engineering and Applied Sciences, \\ Harvard University, Cambridge, MA, USA. \\ ${ }^{2}$ Information and Communications Research Laboratories, \\ Industrial Technology Research Institute (ITRI), Hsinchu, Taiwan.
}

\begin{abstract}
This paper describes a novel data modulation method for ultra-low-power wireless uplink communication between a client device of the internet of things (IoT) and a base station (or an access point). The proposed scheme is dubbed as subcarrier index coordinate expression (SICE), which utilizes the indices of orthogonal frequency division multiplexing (OFDM) subcarriers and waveforms with different phase shifts to represent the information to be sent. SICE is targeted for IoT applications with low data rate requirements, and is especially useful for battery-powered IoT devices demanding lowpower communications, due to its characteristics of low peak to average power ratio (PAPR) and high power efficiency in wireless transmission. Unlike ongoing efforts in IoT wireless communications at standardization organizations which focus on simplifying functionalities of the existing protocols to save power, the technique developed in this paper is a fundamentally new and yet OFDM-compatible physical-layer approach that can drastically reduce power consumption of data transmission.
\end{abstract}

\section{INTRODUCTION}

\section{A. Background}

In responding to the tremendous market potential in the Internet of Things (IoT), many of the communications networking standards are striving to adapt their technologies in order to offer energy-efficient wireless connectivity for IoT applications. Typical examples of IoT devices where lowpower wireless communications schemes are critical include wearables such as smart watches, as well as battery-powered infrastructural nodes for smart monitoring/control. For instance, the standardization forums of 3GPP LTE have initiated the discussions on machine-type communications (MTC) [1], which is envisaged to be an enabling protocol for supporting IoT connectivity in the future releases of LTE. These efforts will allow the existing protocols to handle low-power IoT communications with reduced functionalities [2].

We postulate that for ultra-low-power communication demanded by IoT devices such as coin battery powered sensors, fundamentally new schemes for uplink communications (signaling and data transmission from a client device to the base station) will be required. For example, these schemes will need to exploit aggressively the low data rate property often present in these devices in order to work within stringent resource constraints of low-power, low-memory and low-bandwidth
[3]. This represents a major departure from the mainstream wireless communications research in the last few decades, which has aimed at boosting the achievable data rate, in bid to cope with various high speed mobile applications.

In this work, we study ultra-low-power physical-layer techniques leveraging the popular air-interface based on orthogonal frequency division multiplexing (OFDM) [4], in which the information is transmitted by simultaneously using a large number of orthogonal narrow-band subcarriers. OFDM and its variants have been widely adopted in several important wireless systems standards, including Wi-Fi (IEEE 802.11 a/g/n/ac), 4G cellular networks (3GPP LTE and WiMAX), and terrestrial digital and mobile television. The prevalence of OFDM is mainly attributed to its advantages of high data throughput and robustness to frequency-selective fading channel.

However, for ultra-lower-power IoT uplink communications, we need to overcome an intrinsic problem of OFDM, that is, the potentially high peak to average power ratio (PAPR) [5] of its signal waveform. High PAPR may cause signal distortion and hence performance degradation when the peak of the time-domain waveform (resulted from the sum of a large number of sinusoids with different frequencies associated with OFDM subcarriers) exceeds the linear region of the power amplifier. Although signal distortion caused by non-linearity could be avoided by using expensive high power amplifiers with large dynamic ranges, this is not economical for low-cost IoT devices. Also, the energy efficiency of the power amplifier in transmitters is directly related the PAPR [5]. Thus, the phenomena of high PAPR is particularly undesirable for low-cost and battery-powered IoT devices. There are numerous energy and PAPR reduction algorithms in the existing literature, e.g., [6], [7], but they usually involve closed-loop operations or high-complexity computations that IoT devices cannot afford.

\section{B. Proposed Scheme and Related Work}

We note that a direct approach of reducing power consumption and PAPR is to decrease the number of the activated OFDM subcarriers. Recently, subcarrier-index modulation (SIM) has been proposed in [8], which applies on/off keying (OOK) to the OFDM subcarrier index to convey information. That is, the $N$ subcarriers are dedicated to represent 
$N$ bits of data, and each subcarrier is activated when its correspondent information bit is 1 (or 0 ), and inactivated when its correspondent information bit is 0 (or 1 ). Traditional modulation symbols are further launched on the activated subcarriers to increase the data rate. Since a portion of the subcarriers are switched off, certain amount of transmission energy can be saved. However, subcarrier activation associating to either 1 or 0 is contingent to whether $1 \mathrm{~s}$ or 0 s form the majority in the information bit stream, so at least half of the subcarriers are activated. Also, an extra feedforward signaling is required to notify the receiver which binary number ( 0 s or 1s) is the majority, so the data can be successfully decoded. An enhanced version of SIM has appeared in [9], which dispenses the need of feedforward signal through implication using activation pattern arrangements, but it still requires to switch on at least half of the subcarriers.

In this paper, we put forward a new data modulation method dubbed as subcarrier index information expression (SICE), which presumes information to be transmitted as points of a coordinate system, and utilizes the subcarrier indices as well as waveform phase shifts to modulate the coordinate. For instance, assuming that the information is modeled as an one-dimensional coordinate with positive/negative polarity, where the coordinate value belongs to a set consisting of $2 \mathrm{~N}$ member: $\{ \pm 1, \pm 2, \ldots, \pm N\}$. By simply choosing one subcarrier to activate with a bi-polar signal (positive or negative sinusoid) according to the information coordinate to be sent, while suppressing the other subcarriers, an OFDM system with $N$ subcarriers is able to represent $1+\log _{2} N$ bits of data. That is, the receiver could retrieve the transmitted information by judging which subcarrier is the activated one as well as identifying the associated waveform phase shift. The data rate can be doubled if we additionally switch on a subcarrier (could be the same or a different subcarrier) simultaneously with a different waveform phase shift; this is equivalent to modeling information as points in a two-dimensional system. The data rate can be further increased by using a three-dimensional or even higher-dimensional coordinate system, in which each dimension is designated by a waveform with a distinct phase shift. In contrast to classical OFDM, the number of activated subcarriers with SICE is much fewer. This is advantageous in reducing both power consumption and PAPR. Thus, SICE is an attractive physical-layer uplink signaling technique for low-power, low-cost and low data rate IoT devices.

The rest of the paper is organized as following. A basic review of OFDM technology is provided first. Then, detailed descriptions for both SICE transmitter and receiver algorithms are presented subsequently. In Section $\mathrm{V}$, the benefits of SICE are shown via computer simulation results. Finally, some conclusions and future research directions are given in the end of this paper.

\section{REVIEW OF ORThogonal FREQUENCY Division MuLTIPLEXING (OFDM)}

As aforementioned, SICE piggybacks on the OFDM physical layer ultrastructure. In this section, we provide a brief overview of OFDM and also the issues concerning PAPR.

The basic concept of OFDM involves using a large number (e.g., 64, 128 or higher) of narrow-band subcarriers to transmit information. By using an OFDM system with $N$ subcarriers, we could simultaneously modulate $N$ independent data on different frequencies via inverse fast Fourier transform (IFFT). The basic block diagram for OFDM is depicted in Figure 1. Mathematically, $N$ independent data symbols are allocated among $N$ subcarriers to form an $N \times 1$ frequency-domain signal vector $\boldsymbol{S}_{f}$ :

$$
\boldsymbol{S}_{f}=\left[d_{1} d_{2} \ldots d_{k} \ldots d_{N}\right]^{T}
$$

where $d_{k}, d=1, \ldots, N$, is the data symbol assigned to the $k$-th subcarrier, and is drawn from the alphabet of a certain digital modulation scheme, e.g., quadrature phase shift keying (QPSK). The total data rate is the number of bits each data symbol carrying multiplies the total number of subcarriers used. Then, IFFT is applied on $\boldsymbol{S}_{f}$ to generate a time-domain waveform termed as the OFDM symbol:

$$
\begin{aligned}
\boldsymbol{S}_{t}(\tau) & =\operatorname{IFFT}\left\{\boldsymbol{S}_{f}\right\} \\
& =\frac{1}{N} \sum_{\tau=1}^{N-1} \boldsymbol{S}_{f}(k) \mathrm{e}^{\frac{-j 2 \pi k \tau}{N}}, \quad \tau=0, \ldots, N-1
\end{aligned}
$$

At the output of IFFT, a cyclic prefix (CP) of length $g$ samples is then appended to the beginning of $\boldsymbol{S}_{t}$ as the guard interval between consecutive OFDM symbols, in order to mitigate the effects of inter-symbol interference (ISI) caused by dispersive multipath propagation. The overall time-domain waveform with $N+g$ samples is:

$$
\boldsymbol{S}_{t, c p}(\tau)=\left[\boldsymbol{S}_{t}(N-g+1) \ldots \boldsymbol{S}_{t}(N-1) \boldsymbol{S}_{t}(N)\right]
$$

Clearly, since the time-domain waveform (2) is the sum of $N$ sinusoidal waveforms with different frequencies, it could incur a large peak in wave construction, and hence a high PAPR. The definition of PAPR is:

$$
\text { PAPR }=\frac{\max \left[\left|\boldsymbol{S}_{t, c p}(\tau)\right|^{2}\right]}{\mathrm{E}\left[\left|\boldsymbol{S}_{t, c p}(\tau)\right|^{2}\right]},
$$

where $E[\cdot]$ is the expectation operator. As aforementioned, high PAPR is undesirable for a low-cost IoT device, as the signal peak would easily exceed the linear region of the amplifier and lead to performance degradation in terms of error probabilities and power efficiency.

The main objective of this paper is to report a novel OFDM modulation method that can reduce power consumption and PAPR significantly for low data rate IoT applications. Under the scheme developed in this paper, the activation of the subcarriers is dependent on the information to be sent, and a majority of the entries of $\boldsymbol{S}_{f}$ are expected to be 0 , resulting considerable savings in transmission power. Conversely, classical OFDM schemes would require to activate more subcarriers even under low-rate data transmission, as will be discussed in the later sections. 


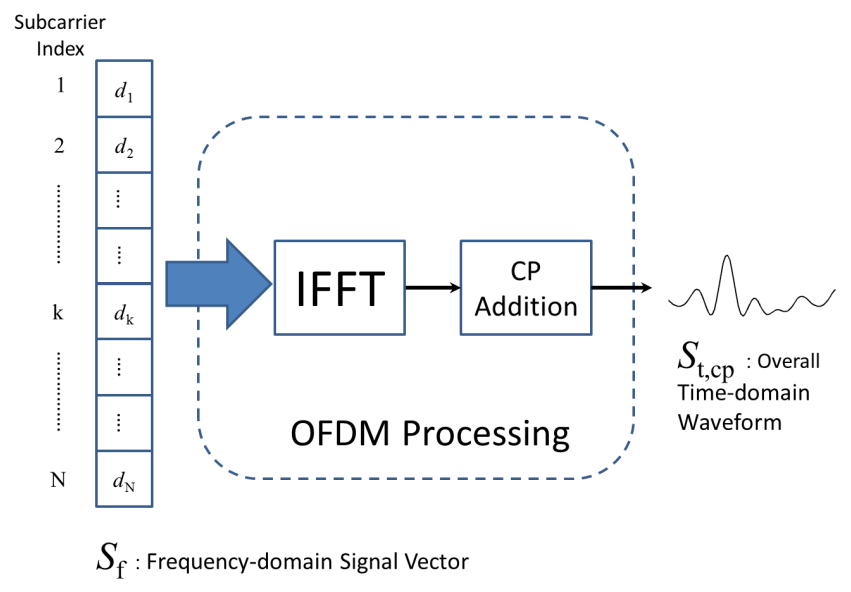

Fig. 1. The basic block diagram of an OFDM-based transmitter.

\section{Detailed Description of SiCE}

\section{A. Information Coordinate Systems}

We view all information to be transmitted as a set $\mathcal{S}$ of points in an $M$-dimensional symmetrical coordinate system. Every member of the set can be written as an $M$-dimensional coordinate: $C_{\mathcal{S}}=\left(c_{1}, \ldots, c_{m}, \ldots, c_{M}\right)$, where the component values are taken from a finite set having cardinality $2 N$ :

$$
c_{1}, \ldots, c_{m}, \ldots, c_{M} \in\left\{ \pm v_{1}, \pm v_{2}, \ldots, \pm v_{N}\right\}
$$

The amount of information that such a coordinate system can convey depends on the set size and the number of dimensions. Specifically, the total number of points in the system: $(2 N)^{M}$. Hence, for an $M$-dimensional coordinated system, the number of bits that are conveyed by one constellation point is:

$$
\begin{aligned}
b & =\log _{2}(2 N)^{M} \\
& =M\left(1+\log _{2} N\right)
\end{aligned}
$$

Figure 2 shows an illustrative example of such coordinate system with $M=2$ and $N=4$ (it may be of interest to note that the structure of this coordinate system coincides with the well-known 64-QAM signal constellation). In this example, every point can be treated as a two-dimensional coordinate $\left(c_{1}, c_{2}\right)$, where the values of both $c_{1}$ and $c_{2}$ are in the set $\{ \pm 1, \pm 3, \pm 5, \pm 7\}$. Also, each of the coordinates conveys 6 bits of information.

\section{B. Details of SICE}

Using the information coordination system described above, we suggest a novel data modulation technique called subcarrier index coordinate expression (SICE). The proposed scheme, incorporated into an OFDM system with $N$ subcarriers, is able to send $b=M\left(1+\log _{2} N\right)$ bits per OFDM symbol. With SICE, the data is modulated via OFDM subcarrier indices and signal waveforms with disparate phase shifts. In particular, each of the $N$ subcarriers is dedicated to indicate the presence of an absolute coordinate component value $\left|v_{n}\right|$, where $n=$ $1, \ldots, N$. The basic schematic of SICE is depicted in Figure 3,

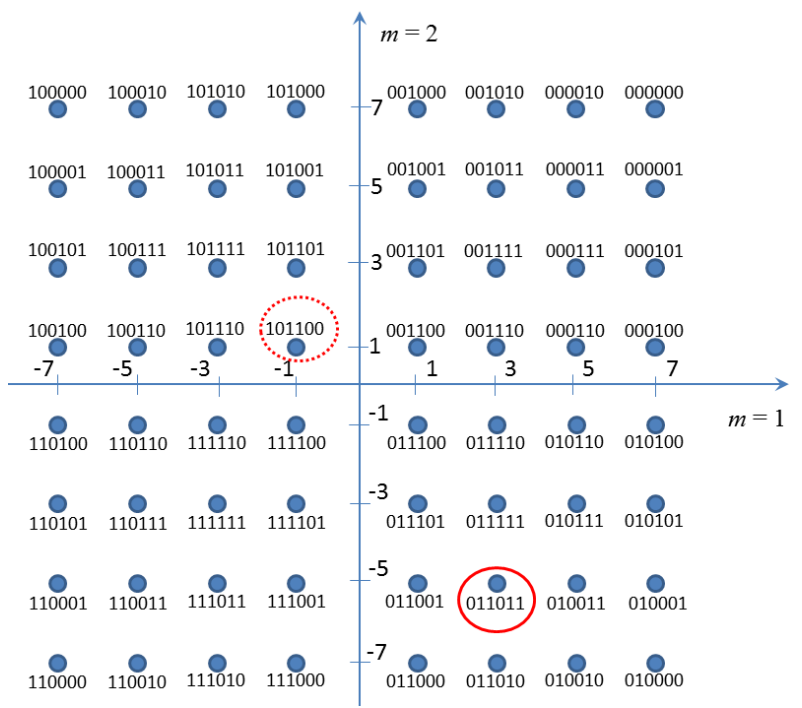

Fig. 2. The 64 points constellation of the two-dimensional information coordinate system $(M=2)$ for SICE using $N=4$ OFDM subcarriers. Two constellation points corresponding to 6-bit blocks '101100' and '011011' are highlighted.

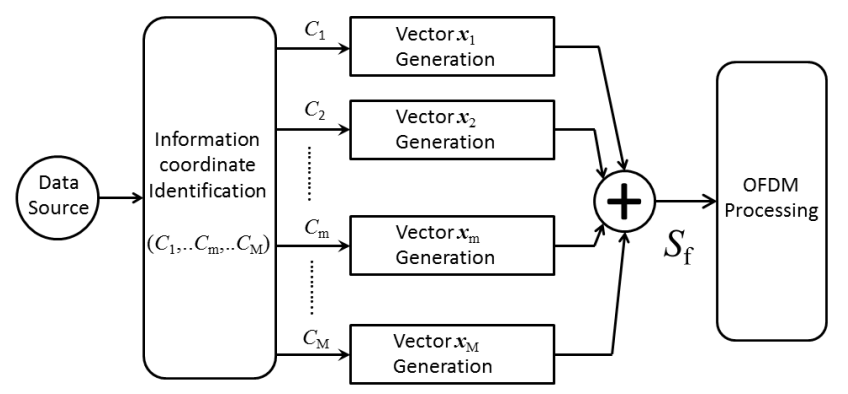

Fig. 3. The schematic of the SICE modulation scheme.

and the detailed operational procedures of SICE is delineated below.

By segmenting the incoming bit stream from the upper layer into data blocks of $b$ bits, the SICE first identifies the $M$ dimensional coordinate for each of the $b$-bit data blocks. The $M$ components of the coordinate are separately mapped to $M N \times 1$ vectors $\boldsymbol{x}_{1}, \ldots, \boldsymbol{x}_{m}, \ldots, \boldsymbol{x}_{M}$, where the entries of each vector, $\boldsymbol{x}_{m}(n), n=1 \ldots N$, respectively corresponding to the $N$ possible absolute coordinate component values $\left\{\left|v_{1}\right|, \ldots,\left|v_{n}\right|, \ldots,\left|v_{N}\right|\right\}$. That is, $\boldsymbol{x}_{m}(1)$ corresponds to $\left|v_{1}\right|$, $\boldsymbol{x}_{m}(2)$ corresponds to $\left|v_{2}\right|$, and so on.

Depending on the sign (positive or negative) of coordinate component in the $m$-th dimension ( $m \in\{1, \ldots, M\}$ ), the entry of vector $\boldsymbol{x}_{m}$ that corresponds to the absolute value of this coordinate component value should be set to either +1 (if this component is positive) or -1 (if this component is negative), while the remaining $N-1$ entries of $\boldsymbol{x}_{m}$ should be set to zero. By undertaking this step on all coordinate components, we can generate $M N \times 1$ vectors, $\boldsymbol{x}_{1}, \ldots, \boldsymbol{x}_{m}, \ldots, \boldsymbol{x}_{M}$. Note that each of the vectors has only one non-zero entry.

Then, all vectors are respectively multiplied by $M$ scalars 
associating to distinct phase shifts:

$$
\overline{\boldsymbol{x}}_{m}=p_{m} \boldsymbol{x}_{m}, \quad m=1, \ldots, M
$$

where

$$
p_{m}=\exp \left(j a_{m} \pi\right), \quad j=\sqrt{-1}, \quad 0 \leq a_{m}<1
$$

and $a_{1} \neq a_{2} \neq \ldots \neq a_{M}$. This is equivalent to labeling each coordinate dimension by a unique phase shift, so the values of $a_{m}$ should not be equal. Otherwise, it would obfuscate the receiver as it cannot determine which dimension this coordinate belongs to. Also, a numerical optimization procedure can be used to find optimal $a_{1}, \ldots, a_{M}$ that minimize PAPR or error probability. Notice that these values should be pre-defined and known at both transmitter and receiver. Finally, the $N \times 1$ frequency-domain signal vector for the OFDM transmission, $\boldsymbol{S}_{f}$, can be constructed by summing up these $M$ vectors:

$$
\boldsymbol{S}_{f}=\sum_{m=1}^{M} \overline{\boldsymbol{x}}_{m}
$$

In a nutshell, the generation of the frequency-domain signal vector $\boldsymbol{S}_{f}$ is tantamount to the matrix operation:

$$
\boldsymbol{S}_{f}=\boldsymbol{X} \boldsymbol{p}
$$

where $\boldsymbol{X}$ is formed by cascading the $M$ column vectors:

$$
\boldsymbol{X}=\left[\boldsymbol{x}_{1}, \ldots, \boldsymbol{x}_{m}, \ldots, \boldsymbol{x}_{M}\right]
$$

and

$$
\boldsymbol{p}=\left[\begin{array}{c}
\exp \left(j a_{1} \pi\right) \\
\vdots \\
\exp \left(j a_{m} \pi\right) \\
\vdots \\
\exp \left(j a_{M} \pi\right)
\end{array}\right]
$$

It is straightforward to see that, at least $N-M$ entries of $\boldsymbol{S}_{f}$ are zero. Therefore, most subcarriers are inactivated since $M$ is much smaller than $N$ in practice. Once the frequency-domain signal vector is ready, the remaining steps are identical to classical OFDM modulation as elucidated in Section II, wherein IFFT and CP addition should be applied to generate the time-domain waveform. Because at least $N-M$ entries of $\boldsymbol{S}_{f}$ in (2) are zero, the time-domain waveform is the summation of merely at most $M$ waveforms with different frequencies. As comparing to classical OFDM, which sums up to $N>>M$ sinusoids to launch the time-domain waveform, SICE can significantly reduce power consumption. Moreover, the resultant time-domain waveform peak with SICE is smaller, and therefore less sensitive to the potential non-linear distortions at the power amplifier.

\section{Examples}

Consider a SICE example with $M=2$ and $N=4$, as shown in Figure 2. Each point is a two-dimensional coordinate $\left(c_{1}, c_{2}\right)$ carrying 6 -bits of information, and the possible values for both abscissa $\left(c_{1}\right)$ and ordinate $\left(c_{2}\right)$ components are taken from the set $\{ \pm 1, \pm 3, \pm 5, \pm 7\}$. This means that $N=4$ subcarriers are required to implement SICE, and these four subcarriers are dedicated to represent absolute coordinate component values $1,3,5$, and 7 respectively. Assuming a 6 -bits information block '011011' is to be sent, the corresponding information coordinate of which (embraced by the solid red circle in Figure 2) can be identified as $\left(c_{1}, c_{2}\right)=(3,-5)$. By following the procedures given in Section III-B, the entry of $\boldsymbol{x}_{1}$ that represents 3 should be set to 1 (since $c_{1}$ is positive), while the remaining entries of $\boldsymbol{x}_{1}$ are all set to 0 . Similarly, the entry of $\boldsymbol{x}_{2}$ that represents 5 should be set to -1 (since $c_{2}$ is negative), while the remaining entries of $\boldsymbol{x}_{2}$ are all set to 0 . Hence, the vectors $\boldsymbol{x}_{1}$ and $\boldsymbol{x}_{2}$ are:

$$
\boldsymbol{x}_{1}=\left[\begin{array}{llll}
0 & 1 & 0 & 0
\end{array}\right]^{T}
$$

and

$$
\boldsymbol{x}_{2}=\left[\begin{array}{llll}
0 & 0 & -1 & 0
\end{array}\right]^{T} .
$$

By setting $a_{1}=0$ and $a_{2}=0.5$, we obtain $p_{1}=$ $\exp \left(j a_{1} \pi\right)=1$ and $p_{2}=\exp \left(j a_{2} \pi\right)=j$. Thus, the frequency-domain signal vector can be computed as

$$
\boldsymbol{S}_{f}=\boldsymbol{X} \boldsymbol{p}=\left[\begin{array}{cc}
0 & 0 \\
1 & 0 \\
0 & -1 \\
0 & 0
\end{array}\right]\left[\begin{array}{l}
1 \\
j
\end{array}\right]=\left[\begin{array}{c}
0 \\
1 \\
-j \\
0
\end{array}\right]
$$

Notice that only two out of the four subcarriers are actually activated. Physically, this is akin to transmitting independent binary phase shift keying (BPSK) signals on two orthogonal subcarriers. With classical OFDM, we could also activate two subcarriers only to send 6 bits in total, by using 8-PSK modulation ( 3 bits per symbol) on each of the two activated subcarriers $(3 \times 2=6)$. Nevertheless, the minimum Euclidean distance between symbols in 8-PSK is obviously smaller than that in BPSK [10], so in theory the resultant error probability would be worse than that of SICE under the same signal to noise ratio (SNR).

Here we inspect another example with $M=2$ and $N=4$ again using the coordinate system depicted in Figure 2. If the transmitter intends to send the information block '101100', the correspondent coordinate can be mapped to $\left(c_{1}, c_{2}\right)=(-1,1)$, which is embraced by the dotted red circle in Figure 2 . Then, by taking the steps of SICE using $a_{1}=0$ and $a_{2}=0.5$, the frequency-domain signal vector is determined as

$$
\boldsymbol{S}_{f}=\boldsymbol{X} \boldsymbol{p}=\left[\begin{array}{cc}
-1 & 1 \\
0 & 0 \\
0 & 0 \\
0 & 0
\end{array}\right]\left[\begin{array}{l}
1 \\
j
\end{array}\right]=\left[\begin{array}{c}
-1+j \\
0 \\
0 \\
0
\end{array}\right]
$$

In this case, only one subcarrier is activated and all the remaining subcarriers are switched off, so the total transmission power is further reduced.

\section{Proposed ReCEIVER Algorithm FOr SICE}

In the time-domain, the received signal model can be written as:

$$
\boldsymbol{Y}_{t, c p}=\boldsymbol{h}_{t} \otimes \boldsymbol{S}_{t, c p}+\boldsymbol{w}
$$


where $\boldsymbol{h}_{t}$ denotes multipath channel response, and $\boldsymbol{w}$ is the additive noise. In order to decode the transmitted data, the receiver should attempt to reverse the transmitter processing and channel effects. Firstly, the $g$ sample points that are deliberately appended as $\mathrm{CP}$ in (3) should be removed:

$$
\boldsymbol{Y}_{t}=\left[\boldsymbol{Y}_{t, c p}(g+1) \ldots \boldsymbol{Y}_{t, c p}(g+N)\right]
$$

Then, fast Fourier transform (FFT) should be applied to acquire the frequency-domain representation of the received signal. That is, $\boldsymbol{Y}_{f}=\operatorname{FFT}\left\{\boldsymbol{Y}_{t}\right\}$. Moreover, the knowledge regarding channel response is assumed to be available at the receiver (this is a reasonable assumption based on common mechanisms such as pilot signals and preambles), so channel equalization can be undertaken as:

$$
\hat{\boldsymbol{S}}_{f}=\frac{\boldsymbol{Y}_{f}}{\operatorname{FFT}\left\{\boldsymbol{h}_{t}\right\}} .
$$

In order to decode the transmitted data, it is essential for the receiver to determine

- The subset of subcarriers that are activated at the transmitter.

- The transmitted waveforms on these activated subcarriers. Based on the property of SICE method, we know that at most only $M$ entries of $\boldsymbol{S}_{f}$ are activated while the other subcarriers are completely switched off. Thus, the receiver can focus on the entries of $\hat{\boldsymbol{S}}_{f}$ with higher signal power, and discard the entries of low signal power by treating them as noise. The receiver first nominates $Q \geq M$ entries of $\hat{\boldsymbol{S}}_{f}$ with the highest power. Then, maximum-likelihood (ML) detection can be carried out via an exhaustive search, which aims to minimize signal space distance between the $Q$ nominated entries of $\hat{\boldsymbol{S}}_{f}$ and all possible signal configurations in the $Q$ corresponding entries of $\boldsymbol{S}_{f}$. Once the transmitted frequencydomain signal vector is determined, it is mapped back to the information coordinate system to extract data bits. The block diagram for the receiver is shown in Figure 4.

It is apparent that the receiver complexity can be increased significantly if $M$ is large. Thus, in bid to limit the receiver complexity and bound PAPR, $M=2$ or 3 could be more appropriate in practice. Also, since the receiver of uplink signaling from IoT devices are generally sophisticated entities with external power source such as base stations or access points, they should be able to accommodate such computational complexity. This is also worth pointing out that, the proposed decoding algorithm is compatible to and yet simpler than conventional ML-based OFDM receivers, as only $Q \leq N$ subcarriers are examined with ML detection. Finally, a high degree of latitude can be excised in SICE receiver design, as the receivers are generally not constrained by protocols.

\section{Simulation Results}

In this section, we present computer simulation results to validate the benefits of SICE as compared to conventional OFDM schemes under the same data rate. In particular, simulations have been carried out based on the following settings and assumptions:

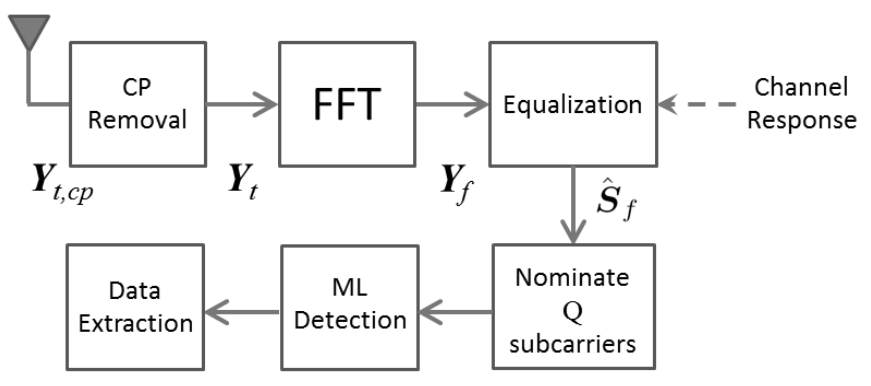

Fig. 4. The receiver processing for SICE modulation scheme.

- The number of subcarriers is $N=64$.

- The CP length is $25 \%$ of the length of IFFT output $\boldsymbol{S}_{t}$.

- Frequency-selective Rayleigh fading channel with 6-taps is adopted for simulations. Each channel impulse responses coefficient (the elements of $\boldsymbol{h}_{t}$ ) is modeled as a circularly symmetric complex Gaussian random variable eN $\left(0, \frac{1}{6}\right)$.

- The power allocation per activated subcarrier is $\frac{1}{N}$.

We first compare the data error probabilities. The settings for classical OFDM and SICE are:

1) With classical OFDM, QPSK (2-bits) is applied on 7 subcarriers.

2) With SICE, the parameters $M=2, a_{1}=0$ and $a_{2}=0.5$ are used.

Figure 5 presents the comparisons of OFDM symbol error probabilities. Under a data rate of 14 bits per OFDM symbol interval, SICE with $M=2$ outperforms classical OFDM when the signal to noise ratio (SNR) is sufficiently high. In terms of power consumption, classical OFDM activates 7 subcarriers to transmit 14 bits of information, while SICE only requires to activate at most 2 subcarriers. Since we have assumed that power allocation per activated subcarrier is the same, the power consumption for SICE is only $2 / 7$ (or $28.57 \%$ ) of that for classical OFDM. As mentioned earlier, one could argue that higher-order modulation schemes can be used on classical OFDM to reduce the number of activated subcarriers; however, theoretically it would deteriorate error probability under the same SNR level, since boosting modulation order is equivalent to decreasing the minimum Euclidean distance between data signals.

The complementary cumulative distribution function (CCDF) for PAPR, which measures the probability of PAPR being larger than a threshold level $\Gamma$, is used to compare the PAPR of SICE and that of classical OFDM in Figure 6. In this context, we also added the results of SICE with $M=3$ and classical OFDM with 8-PSK on 7 subcarriers for further comparison, both of these schemes are transmitting 21 bits per OFDM symbol. For SICE cases, the probability of PAPR larger than $\Gamma$ decays much quicker than the classical OFDM schemes. This means that the resultant PAPR of SICE is generally lower than that of classical OFDM, and such property directly relaxes the requirements on linear region of power amplifiers. These simulation results have demonstrated 


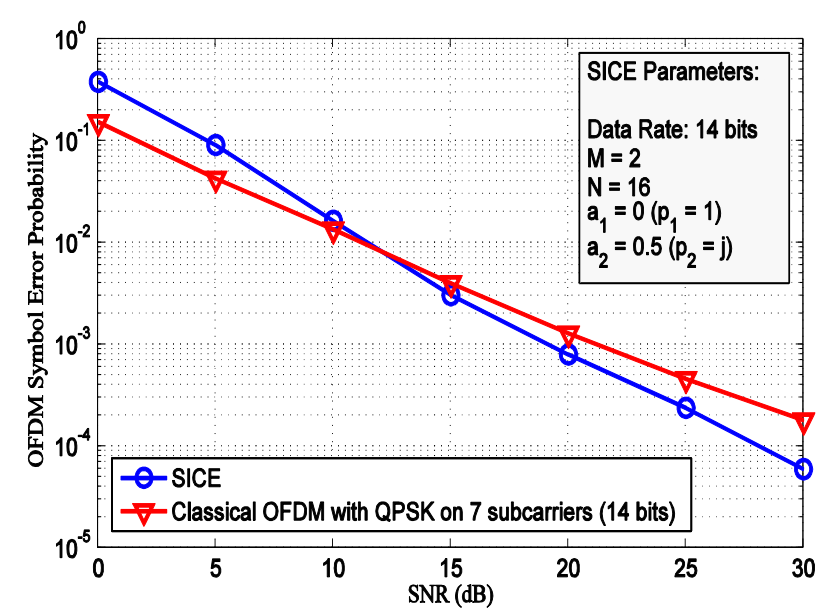

Fig. 5. The comparison of data error probability for SICE and classical OFDM under the same data rate: 14 bits per OFDM symbol interval.

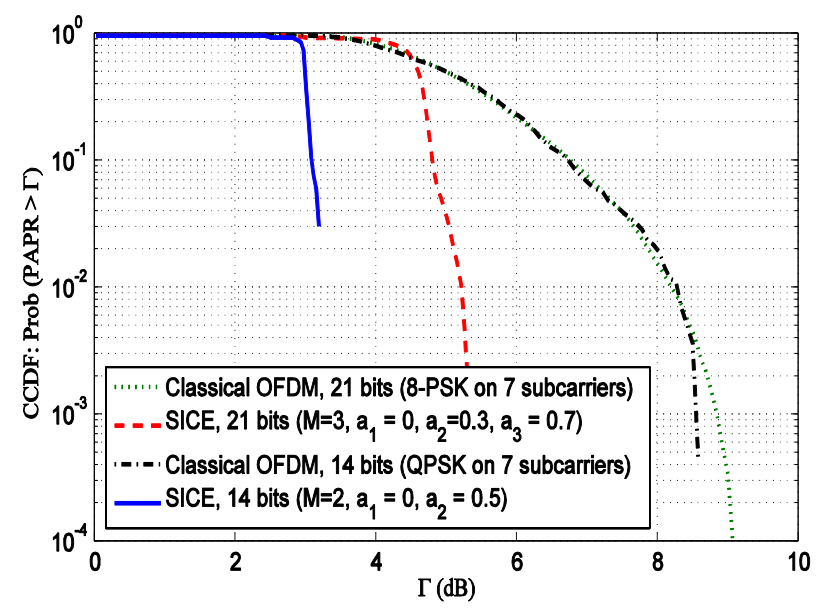

Fig. 6. The comparison of CCDF for PAPR between SICE and classical OFDM under the same data rate.

benefits of SICE in reliability, power efficiency, and PAPR characteristics.

\section{CONCLUSiOnS AND Future Work}

This paper proposed SICE as a novel data transmission scheme which piggybacks on the existing OFDM physical layer ultrastructure. Based on the following reasons, we can conclude that SICE is suitable for uplink wireless communications of IoT devices:

- SICE is designed to operate within the OFDM framework, and OFDM is the physical-layer technique that has been adopted for Wi-Fi and 4G; both of these networking protocols have the enormous potential to play key roles in offering wireless connectivity for IoT [11].

- Under the same data rate, SICE activates fewer subcarriers than classical OFDM. This directly improves power efficiency and thereby lengthens battery life of IoT devices, while maintaining similar, or even better, data transmission reliability. Such property also results in a low PAPR of the time-domain waveform, so the OFDM signal can be launched in the linear region of a low-cost power amplifier in IoT devices.

- Although the achievable peak data rate of SICE is not as high as classical OFDM under the same amount of available subcarriers $N$, this is not a critical issue since high data rate is not needed in uplink scenarios for lowcost and low-power IoT devices [12].

For the future research directions, approaches of increasing data rate of SICE are worth exploring. For instance, one may use a specific subset of subcarriers (instead of only one) to represent the absolute coordinate component value, so the data rate could be boosted by exploiting combinational gain. On the other hand, optimization of phase shifts parameters $a_{1}, \ldots, a_{M}$ to further reduce error probability and PAPR is another issue that should be addressed.

Finally, analogous to the Wireless Inference-based Notification (WIN) protocol for sensor networks [13], SICE can be considered as an example of low-power communications schemes that convey information using radio resource activation patterns. We believe this is a promising trend for the paradigm of energy-efficient wireless communications for IoT devices.

\section{REFERENCES}

[1] 3GPP, "Study on provision of low-cost machine-type communications (MTC) user equipments (UEs) based on LTE," TSG RAN," TR 36.888 V12.0.0, June 2013.

[2] WG802.16p, IEEE Standard for Air Interface for Broadband Wireless Access Systems - Amendment 1: Enhancements to Support Machine-toMachine Applications, Std., 2012.

[3] F. daCosta, Rethinking the internet of things: A scalable approach to connecting everything. Apress, 2014.

[4] T. Hwang, C. Yang, G. Wu, S. Li, and G. Y. Li, "OFDM and its wireless applications: A survey," IEEE Trans. Veh. Technol., vol. 58, pp. 16731694, May 2009.

[5] G. Wunder, R. F. H. Fischer, H. Boche, S. Litsyn, and J.-S. No, "The PAPR problem in OFDM transmission: New directions for a long-lasting problem," IEEE Signal Processing Mag., vol. 30, pp. 130-144, Nov. 2013.

[6] C. Y. Wong, R. S. Cheng, K. B. Letaief, and R. D. Murch, "Multiuser OFDM with adaptive subcarrier, bit, and power allocation," IEEE $J$. Select. Areas Commun., vol. 17, pp. 1747-1758, Oct. 1999.

[7] T. Jiang and Y. Wu, "An overview: Peak-to-average power ratio reduction techniques for OFDM signals," IEEE Trans. Broadcast., vol. 54, pp. 257-268, June 2008.

[8] R. Abu-alhiga and H. Haas, "Subcarrier-index modulation OFDM," in Proc. IEEE Int. Symp. on Personal, Indoor, Mobile Radio Communications (PIMRC), Tokyo, Japan, Sept. 2009, pp. 177-181.

[9] D. Tsonev, S. Sinanovic, and H. Haas, "Enhanced subcarrier-index modulation (SIM) OFDM," in Proc. IEEE Globecom Workshops, Houston, TX, USA, Dec. 2011, pp. 728-732.

[10] J. Proakis and M. Salehi, Digital Communications, 5th ed. McGraw Hill, 2007.

[11] J. Gubbia, R. Buyyab, S. Marusica, and M. Palaniswamia, "Internet of things (IoT): A vision, architectural elements, and future directions," Future Generation Computer Systems, vol. 29, pp. 1645-1660, Sept. 2013.

[12] U. Raza, A. L. Murphy, and G. P. Picco, "Pushing a standard wireless sensor network stack for ultra-low data rates," in Proc. ACM Conf. on Embedded Networked Sensor Systems (SenSys), Rome, Italy, Nov. 2013.

[13] H.-C. Chen and H. T. Kung, "Wireless inference-based notification (WIN) without packet decoding," in Proc. Int. Conf. on Autonomic Computing (ICAC), San Jose, CA, USA, June 2013, pp. 325-330. 